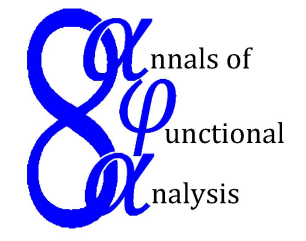

Ann. Funct. Anal. 5 (2014), no. 1, 24-29

$\mathscr{A}$ NNALS OF $\mathscr{F}$ UNCTIONAL $\mathscr{A}$ NALYSIS

ISSN: 2008-8752 (electronic)

URL:www.emis.de/journals/AFA/

\title{
AN OPERATOR INEQUALITY IMPLYING THE USUAL AND CHAOTIC ORDERS
}

\author{
JUN ICHI FUJII*1, MASATOSHI FUJII² AND RITSUO NAKAMOTO ${ }^{3}$ \\ Dedicated to Professor Tsuyoshi Ando for his significant contributions to our areas \\ Communicated by Y. Seo
}

AbStract. We prove that if positive invertible operators $A$ and $B$ satisfy an operator inequality $\left(B^{s / 2} A^{(s-t) / 2} B^{t} A^{(s-t) / 2} B^{s / 2}\right)^{\frac{1}{2 s}} \geq B$ for some $t>s>0$, then

(1) If $t \geq 3 s-2 \geq 0$, then $\log B \geq \log A$, and if $t \geq s+2$ is additionally assumed, then $B \geq A$.

(2) If $0<s<1 / 2$, then $\log B \geq \log A$, and if $t \geq s+2$ is additionally assumed, then $B \geq A$.

It is an interesting application of the Furuta inequality. Furthermore we consider some related results.

\section{INTRODUCTION}

An operator means a bounded linear operator acting on a Hilbert space. The usual order $A \geq B$ among selfadjoint operators on $H$ is defined by $(A x, x) \geq$ $(B x, x)$ for any $x \in H$. In particular, $A$ is said to be positive and denoted by $A \geq 0$ if $(A x, x) \geq 0$ for $x \in H$, and $A>0$ if $A$ is invertible.

The noncommutativity of operators reflects on the usual order, [8] and [13], as follows:

\section{Löwner-Heinz inequality:}

$$
A \geq B \Rightarrow A^{p} \geq B^{p}
$$

if and only if $p \in[0,1]$.

Date: Received: 20 March 2013; Accepted: 28 April 2013.

* Corresponding author.

2010 Mathematics Subject Classification. Primary 47A63; Secondary, 47A56.

Key words and phrases. Operator inequality, chaotic order, Furuta inequality. 
In 1987, Furuta [6] proposed a beautiful extension of (LH), by which the restriction $p \in[0,1]$ in $(\mathrm{LH})$ is relaxed in some sense:

Furuta inequality: If $A \geq B$, then for each $r \geq 0$,

$$
\left(A^{r / 2} B^{p} A^{r / 2}\right)^{\frac{1}{q}} \leq A^{\frac{p+r}{q}} \quad \text { and } \quad B^{\frac{p+r}{q}} \leq\left(B^{r / 2} A^{p} B^{r / 2}\right)^{\frac{1}{q}}
$$

hold for $p \geq 0$ and $q \geq 1$ with

$$
(1+r) q \geq p+r
$$

For the Furuta inequality, we refer [2],[5],[6] and [7]. Among others, the best possibility of the domain determined by $(\dagger)$ is proved by Tanahashi [14].

Afterwards, the Furuta inequality was discussed under the chaotic order $\log A \geq$ $\log B$ for $A, B>0$, which was originally discussed by Ando [1], and the final result was obtained in [3].

Theorem FFK. The following (1) - (3) are mutually equivalent for $A, B>0$ :

(1) $\log A \geq \log B$

(2) $A^{p} \geq\left(A^{p / 2} B^{p} A^{p / 2}\right)^{1 / 2}$ for $p \geq 0$,

(3) $A^{r} \geq\left(A^{r / 2} B^{p} A^{r / 2}\right)^{\frac{r}{p+r}}$ for $p, r \geq 0$.

From the viewpoint of Kamei's satellite theorem [12] and Uchiyama's work [15], we here mention that Theorem FFK is equivalent to the Furuta inequality.

Now we consider the following operator inequality for positive invertible operators $A$ and $B$ :

$$
\left(B^{s / 2} A^{(s-t) / 2} B^{t} A^{(s-t) / 2} B^{s / 2}\right)^{\frac{1}{2 s}} \geq B .
$$

Recently, as an application of the Daleckii-Krein formula (see [2]) for the derivative of matrix valued function, one of the authors [4] proved that if matrices $A, B$ satisfy $(*)$ for any $t>1$ and $s=1$, then $\log B \geq \log A$. In this situation, recalling the equivalence between Theorem FFK and the Furuta inequality, it is expected that the conclusion $\log B \geq \log A$ is built up the usual order $B \geq A$.

In this note, we prove that if positive operators $A$ and $B$ satisfy the operator inequality $(*)$ for a fixed $t>s>0$, then

(1) If $t \geq 3 s-2 \geq 0$, then $\log B \geq \log A$, and if $t \geq s+2$ is additionally assumed, then $B \geq A$.

(2) If $0<s<1 / 2$, then $\log B \geq \log A$, and if $t \geq s+2$ is additionally assumed, then $B \geq A$.

\section{A PRELIMINARY RESUlt FOR THE CHAOTIC ORDER}

In the consideration on Kamei's satellite theorem [12] of the Furuta inequality, we are required some operator inequalities of Furuta type implying the chaotic order. Consequently one of the authors announced the following result in [4]: If positive definite matrices $A, B>0$ satisfy

$$
\left(B^{1 / 2} A^{(1-t) / 2} B^{t} A^{(1-t) / 2} B^{1 / 2}\right)^{1 / 2} \geq B \quad \text { for all } t>1,
$$

then $\log B \geq \log A$. 
We now generalize it as follows:

Theorem 1. For positive definite matrices $A, B>0$, if there exist $\alpha, \beta$ such that $\alpha+\beta=1$ and

$$
\left(B^{(\alpha+\beta t) / 2} A^{(1-t) / 2} B^{\beta+\alpha t} A^{(1-t) / 2} B^{(\alpha+\beta t) / 2}\right)^{1 / 2} \geq B \quad \text { for all } t>1,
$$

then $\log B \geq \log A$.

Proof. Let $F(x)=x^{1 / 2}, \gamma(t)=B^{(\alpha+\beta t) / 2} A^{(1-t) / 2} B^{\beta+\alpha t} A^{(1-t) / 2} B^{(\alpha+\beta t) / 2}$ and $U_{t}$ be unitaries such that $U_{t}^{*} \gamma(t) U_{t}=D(t)=\operatorname{diag}\left(d_{1}(t), \cdots, d_{n}(t)\right)$, diagonal matrices. Here we recall the Daleckii-Krein formula

$$
\frac{d F(\gamma(t))}{d t}=U_{t}\left(\left(F^{[1]}\left(d_{i}(t), d_{j}(t)\right)\right) \circ U_{t}^{*} \dot{\gamma}(t) U_{t}\right) U_{t}^{*}
$$

where $\circ$ stands for the Hadamard-Schur product and $F^{[1]}(x, y)$ is the divided difference

$$
F^{[1]}(x, y)=\left\{\begin{array}{ll}
\frac{F(x)-F(y)}{x-y} & \text { if } x \neq y \\
F^{\prime}(x) & \text { if } x=y
\end{array} .\right.
$$

We may assume that $B$ itself is a diagonal matrix $\operatorname{diag}\left(d_{j}\right)$, so $U_{1}=I$, the identity matrix. Therefore, at $t=1$, we obtain

$$
\frac{d F(\gamma)}{d t}(1)=\left(F^{[1]}\left(d_{i}^{2}, d_{j}^{2}\right)\right) \circ \dot{\gamma}(1) \text { and }\left(F^{[1]}\left(d_{i}^{2}, d_{j}^{2}\right)\right)=\left(\frac{d_{i}-d_{j}}{d_{i}^{2}-d_{j}^{2}}\right)=\left(\frac{1}{d_{i}+d_{j}}\right) \text {. }
$$

It follows that

$$
\begin{aligned}
\dot{\gamma}(t) & =\frac{\beta}{2}(\log B) B^{(\alpha+\beta t) / 2} A^{(1-t) / 2} B^{\beta+\alpha t} A^{(1-t) / 2} B^{(\alpha+\beta t) / 2} \\
& +\alpha B^{(\alpha+\beta t) / 2} A^{(1-t) / 2}(\log B) B^{\beta+\alpha t} A^{(1-t) / 2} B^{(\alpha+\beta t) / 2} \\
& +\frac{\beta}{2} B^{(\alpha+\beta t) / 2} A^{(1-t) / 2} B^{\beta+\alpha t} A^{(1-t) / 2} B^{(\alpha+\beta t) / 2}(\log B) \\
& -\frac{1}{2} B^{(\alpha+\beta t) / 2}(\log A) A^{(1-t) / 2} B^{\beta+\alpha t} A^{(1-t) / 2} B^{(\alpha+\beta t) / 2} \\
& -\frac{1}{2} B^{(\alpha+\beta t) / 2} A^{(1-t) / 2} B^{\beta+\alpha t}(\log A) A^{(1-t) / 2} B^{(\alpha+\beta t) / 2} \\
\dot{\gamma}(1) & =(\log B) B^{2}-\frac{1}{2} B^{1 / 2}(\log A) B^{3 / 2}-\frac{1}{2} B^{3 / 2}(\log A) B^{1 / 2} \\
& =\frac{1}{2} B^{1 / 2}(B(\log B-\log A)+(\log B-\log A) B) B^{1 / 2} \\
& =\frac{1}{2}\left(\mathbf{L}_{B}+\mathbf{R}_{B}\right)\left(B^{1 / 2}(\log B-\log A) B^{1 / 2}\right) \\
& =\frac{1}{2}\left(\left(d_{i}+d_{j}\right)\right) \circ\left(B^{1 / 2}(\log B-\log A) B^{1 / 2}\right)
\end{aligned}
$$


as $t \longrightarrow 1$, so we have

$$
\begin{aligned}
\frac{d F(\gamma)}{d t}(1) & =\left(F^{[1]}\left(d_{i}, d_{j}\right)\right) \circ \dot{\gamma}(1) \\
& =\left(\frac{1}{d_{i}+d_{j}}\right) \circ\left(\frac{1}{2}\left(\left(d_{i}+d_{j}\right)\right) \circ\left(B^{1 / 2}(\log B-\log A) B^{1 / 2}\right)\right) \\
& =\frac{1}{2}\left(B^{1 / 2}(\log B-\log A) B^{1 / 2}\right) .
\end{aligned}
$$

On the other hand, since

$$
\frac{d F(\gamma)}{d t}(1)=\lim _{t \downarrow 1} \frac{\left(B^{(\alpha+\beta t) / 2} A^{(1-t) / 2} B^{\beta+\alpha t} A^{(1-t) / 2} B^{(\alpha+\beta t) / 2}\right)^{1 / 2}-B}{t-1} \geq 0,
$$

we obtain $B^{1 / 2}(\log B-\log A) B^{1 / 2} \geq 0$, that is, $\log B \geq \log A$.

\section{MAin TheOREMS}

The operator inequality $(*)$ is a multiple version of the Furuta inequality. We here generalize Theorem 1 to the case with 2 variables. Nevertheless, the Furuta inequality is applicable to resolve it. In this section, we first propose the following theorem.

Theorem 2. Suppose that $A, B>0$ satisfy the inequality (*), i.e.,

$$
\left(B^{s / 2} A^{(s-t) / 2} B^{t} A^{(s-t) / 2} B^{s / 2}\right)^{\frac{1}{2 s}} \geq B
$$

for some $t>s>0$. Then the following assertions hold:

(1) If $t \geq 3 s-2 \geq 0$, then $\log B \geq \log A$, and if the additional condition $t \geq s+2$ is assumed, then $B \geq A$.

(2) If $0<s<1 / 2$, then $\log B \geq \log A$, and if the additional condition $t \geq s+2$ is assumed, then $B \geq A$.

Proof. By the Furuta inequality, we have for $p=2 s$ and $r=t-s$

$$
\left(B^{(t-s) / 2} B^{s / 2} A^{(s-t) / 2} B^{t} A^{(s-t) / 2} B^{s / 2} B^{(t-s) / 2}\right)^{\frac{t-s+1}{t+s}} \geq B^{t-s+1}
$$

that is,

$$
\left(B^{t / 2} A^{(s-t) / 2} B^{t} A^{(s-t) / 2} B^{t / 2}\right)^{\frac{t-s+1}{t+s}} \geq B^{t-s+1} .
$$

Hence we have

$$
\left(B^{t / 2} A^{(s-t) / 2} B^{t / 2}\right)^{\frac{2(t-s+1)}{t+s}} \geq B^{t-s+1} .
$$

Now we prove (1): As $\frac{2(t-s+1)}{t+s}>1$ by $t \geq 3 s-2, B^{t / 2} A^{(s-t) / 2} B^{t / 2} \geq B^{\frac{t+s}{2}}$, and so $A^{(s-t) / 2} \geq B^{(s-t) / 2}$. Consequently, we have $\log B \geq \log A$ by $t>s$ and the operator monotonicity of the logarithmic function. Moreover, if $t \geq s+2$, then $(t-s) / 2 \geq 1$ and so $B \geq A$ by the Löwner-Heinz theorem.

Next, if $s<1 / 2$, then by the Löwner-Heinz inequality, we have

$$
B^{s / 2} A^{(s-t) / 2} B^{t} A^{(s-t) / 2} B^{s / 2} \geq B^{2 s} \text {. }
$$

Hence it follows that $A^{(s-t) / 2} B^{t} A^{(s-t) / 2} \geq B^{s}$ and thus

$$
B^{t / 2} A^{(s-t) / 2} B^{t} A^{(s-t) / 2} B^{t / 2} \geq B^{s+t},
$$


that is, $\left(B^{t / 2} A^{(s-t) / 2} B^{t / 2}\right)^{2} \geq B^{s+t}$. Consequently, we have $A^{(s-t) / 2} \geq B^{(s-t) / 2}$ and the conclusion is obtained as in the proof of (1).

Corollary 3. If $A, B>0$ satisfy

$$
\left(B^{1 / 2} A^{(1-t) / 2} B^{t} A^{(1-t) / 2} B^{1 / 2}\right)^{\frac{1}{2}} \geq B \quad \text { for a fixed } t>1
$$

then $\log B \geq \log A$. Moreover if it satisfied for some $t \geq 3$, then $B \geq A$.

Unfortunately the converse in Theorem 2 does not hold.

Example 4. Let $A=\left(\begin{array}{ll}2 & 1 \\ 1 & 1\end{array}\right), B=\left(\begin{array}{ll}3 & 0 \\ 0 & 2\end{array}\right), s=1$ and $t=4$. Then $B \geq A$ and

$$
\sigma\left(\left(B^{1 / 2} A^{(1-t) / 2} B^{t} A^{(1-t) / 2} B^{1 / 2}\right)^{1 / 2}-B\right)=\{35.2421,-0.25003\} .
$$

Theoretically, if operators $A$ and $B$ satisfy $(*)$, then $B^{(t-1) / 2} \geq A^{(t-1) / 2}$ as in the proof of Theorem 2. However in general, $B \geq A$ does not imply $B^{(t-1) / 2} \geq$ $A^{(t-1) / 2}$, Hence $B \geq A$ does not always imply $(*)$.

Remark 5. It must be $t>1$ in order to imply $B \geq A$. If $A$ commutes with $B$, then we have $\left(A^{1-t} B^{1+t}\right)^{1 / 2} \geq B$, that is, $B^{t-1} \geq A^{t-1}$. Hence $B \geq A$ if $t>1$.

For $1>t>0$, we prove the following theorem by applying Lyapunov equation, see [2] and [9].

Theorem 6. If $A, B>0$ satisfy $(*)$ for $s=1$ and any $t \in(0,1)$, then $\log A \geq$ $\log B$.

Proof. Put $X_{t}=B^{1 / 2} A^{(1-t) / 2} B^{t} A^{(1-t) / 2} B^{1 / 2}$. Then we have

$$
\left(X_{t}^{1 / 2}-B\right) X_{t}^{1 / 2}+B\left(X_{t}^{1 / 2}-B\right)=X_{t}-B^{2}
$$

and

$$
\begin{aligned}
& X_{t}-B^{2}= \\
& B^{1 / 2}\left\{A^{(1-t) / 2}\left(B^{t}-B\right) A^{(1-t) / 2}+A^{(1-t) / 2} B\left(A^{(1-t) / 2}-1\right)+\left(A^{(1-t) / 2}-1\right) B\right\} B^{1 / 2} .
\end{aligned}
$$

Here we have

$$
\lim _{t \rightarrow 1} \frac{B^{t}-B}{t-1}=B \log B \text { and } \lim _{t \rightarrow 1} \frac{A^{(1-t) / 2}-1}{t-1}=-\frac{1}{2} \log A .
$$

Hence by putting $Y=\lim _{t \rightarrow 1} \frac{X_{t}^{1 / 2}-B}{t-1}$ via the chain rule (cf. [11, Theorem 8.4]), it follows that $Y \leq 0$ by the assumption and

$$
B Y+Y B=B^{1 / 2}\left(B \log B-\frac{1}{2}(B \log A+\log A B)\right) B^{1 / 2} .
$$

By solving this Lyapunov equation,

$$
\begin{aligned}
Y & =B^{1 / 2}\left(\int_{-\infty}^{0} e^{t B}\left(\left(B \log B-\frac{1}{2}(B \log A+\log A B)\right) e^{t B} d t\right) B^{1 / 2}\right. \\
& =B^{1 / 2}\left(\frac{1}{2} \log B\left[e^{2 t B}\right]_{-\infty}^{0}-\frac{1}{2}\left[e^{t B} \log A e^{t B}\right]_{-\infty}^{0}\right) B^{1 / 2} \\
& =\frac{1}{2} B^{1 / 2}(\log B-\log A) B^{1 / 2}
\end{aligned}
$$


Since $Y \leq 0$, we have $\log A \geq \log B$.

\section{REFERENCES}

1. T. Ando, On some operator inequality, Math. Ann. 279 (1987), 157-159.

2. R. Bhatia, Positive Definite Matrices, Princeton Univ. Press, 2007.

3. M. Fujii, Furuta's inquality and its mean theoretic approach, J. Operator Theory 23 (1990), $67-72$.

4. M. Fujii, T. Furuta and E. Kamei, Furuta's inequality and its application to Ando's theorem, Linear Algebra Appl. 179(1993), 161-169.

5. J.I. Fujii, On the Daleckii-Krein differential formula of matrices (in Japanese), The Bulletin of International Society for Mathematical Sciences, no. 83 (2012), 6-11.

6. T. Furuta, $A \geq B \geq 0$ assures $\left(B^{r} A^{p} B^{r}\right)^{1 / q} \geq B^{(p+2 r) / q}$ for $r \geq 0, p \geq 0, q \geq 1$ with $(1+2 r) q \geq p+2 r$, Proc. Amer. Math. Soc. 101 (1987), 85-88.

7. T. Furuta, An elementary proof of an order preserving inequality, Proc Japan Acad. 65 (1989), 126.

8. T. Furuta, Invitation to Linear Operators, Taylor and Francis, 2001.

9. E. Heinz, Beitrage zur Störungstheorie der Spektralzerlegung, Math. Ann. 123 (1951), 415438.

10. R.A. Horn and C.R. Johnson, Topics in Matrix Analysis, Cambridge Univ. Press, 1991.

11. J. Jost, Postmodern Analysis 3rd ed., Springer, 2005.

12. E. Kamei, A satellite to Furuta's inequality, Math. Japon. 33 (1988), 833-836.

13. K. Löwner, Über monotone Matrixfunctionen, Math. Z. 38 (1934), 177-216.

14. K. Tanahashi, Best possibility of the Furuta inequality, Proc. Amer. Math. Soc. 128 (1996), $141-146$.

15. M. Uchiyama, Some exponential operator inequalities, Math. Inequal. Appl. 2 (1999), 469471.

${ }^{1}$ Department of Arts and Sciences (Information Science), Osaka Kyoiku UniVERSITY, KashiWARA, OSAKa 582-8582, JaPAN.

E-mail address: fujii@cc.osaka-kyoiku.ac.jp

2 Department of Mathematics, Osaka Kyoiku University, Kashiwara, Osaka 582-8582, JAPAN.

E-mail address: mfujii@cc.osaka-kyoiku.ac.jp

3 1-4-13, Daihara-cho, Hitachi, Ibaraki 316-0021, Japan.

E-mail address: r-naka@net1.jway.ne.jp 